Review Article

\title{
Prognostic Value of Frailty for Older Patients with Heart Failure: A Systematic Review and Meta-Analysis of Prospective Studies
}

\author{
Xige Wang $\mathbb{D}^{D}$, Changli Zhou, Yuewei Li, Huimin Li $\mathbb{D}$, Qinqin Cao $\mathbb{D}$, and Feng Li $\mathbb{D}$ \\ School of Nursing, Jilin University, 965 Xinjiang Street, Changchun, Jilin 130020, China \\ Correspondence should be addressed to Feng Li; fli@jlu.edu.cn
}

Received 10 July 2018; Revised 13 September 2018; Accepted 9 October 2018; Published 22 October 2018

Academic Editor: Hiroyuki Shimada

Copyright (c) 2018 Xige Wang et al. This is an open access article distributed under the Creative Commons Attribution License, which permits unrestricted use, distribution, and reproduction in any medium, provided the original work is properly cited.

\begin{abstract}
Objective. Numerous studies have investigated the prognostic role of frailty in elderly patients with heart failure (HF), but the limited size of the reported studies has resulted in continued uncertainty regarding its prognostic impact. The aim of this study was to integrate the findings of all available studies and estimate the impact of frailty on the prognosis of HF by performing a systematic review and meta-analysis. Methods. PubMed, Embase, Cochrane, and Web of Science databases were searched from inception to November $8^{\text {th }} 2017$ to identify eligible prospective studies. The Newcastle-Ottawa Scale (NOS) was used to evaluate study quality. The association between frailty and HF outcomes was reviewed. Overall hazard ratios (HRs) for the effects of frailty on all-cause mortality were pooled using a fixed-effect model and publication bias was evaluated using funnel plots. Results. A total of 10 studies involving 3033 elderly patients with HF were included in the systematic review and meta-analysis. All eligible studies indicated that frailty was of prognostic significance for HF patients. The HRs for the effects of frailty on all-cause mortality were 1.70 (95\% confidence interval (CI): 1.41-2.04), based on the pooling of six studies that provided related data. However, publication bias was observed among the studies. Conclusions. Frailty has a high prevalence among older patients with HF. Elderly HF patients with frailty have a poorer prognosis than those without frailty. Further studies are now required to implement the use of frailty assessment tools and explore effective interventions for frailty in older HF patients.
\end{abstract}

\section{Introduction}

Due to the increased age of the general population, HF patients are predominantly elderly $[1,2]$. HF is the most common cause of hospitalization in the older population leading to a high risk of mortality, disability, and hospital readmission $[3,4]$. Due to poor prognosis and high costs of treatment, adequate risk assessments and optimized treatment decisions are essential in older patients with HF [5]. In the past, the assessment of care for HF patients was primarily based on biological age and subjective symptoms. However, the health trajectory of the elderly is complex and cannot simply be interpreted by physical age criteria or a single disease-specific risk factor [6]. These indicators do not reflect the actual physical health status of older patients, and so current assessments do not fully explain the risk of HF in the elderly $[7,8]$.
Similar to HF, frailty is a geriatric syndrome characterised by decreased physiological activity of multiple organ systems as a result of aging. Frail elderly individuals are prone to adverse events, including falls and hospitalization [9-11]. In recent years, as research on frailty has increased, a high incidence has been identified in HF patients, increasing the risk of adverse outcomes $[12,13]$. This may reflect the central role of the heart in the perfusion of all organs throughout the body and the diffuse damage caused by HF. It may also reflect the common pathophysiological links between HF and frailty $[14,15]$.

Despite numerous studies showing an adverse prognostic impact of frailty on clinical outcomes including survival, the limited size of the individual studies has resulted in persisting uncertainty regarding the prognostic impact of frailty in the older HF population. We therefore performed a systematic review and meta-analysis to integrate the findings of available 
studies and estimate the prognostic value of frailty for older patients with HF.

\section{Methods}

We performed a systematic review and meta-analysis of prospective studies to estimate the prognostic value of frailty for older patients with HF. This systematic review and metaanalysis was performed according to PRISMA guidelines.

2.1. Eligibility Criteria. Studies were required to meet all of the following inclusion criteria: (1) patients: subjects with $\mathrm{HF}$, age $\geq 65$ years; (2) interventions: subjects with frailty; (3) comparators: subjects without frailty; (4) outcomes: allcause mortality or all-cause hospitalization or HF-related hospitalization. Hazard ratios (HRs) with 95\% confidence intervals (CI) had to be included.

2.2. Information Sources. PubMed, Embase, Cochrane, and the Web of Science databases, from inception to November 8th 2017, were searched for relevant articles.

2.3. Search Criteria. The search strategy included Mesh terms for "Frail", "Elderly", and "Heart Failure". The language used in included manuscripts was limited to English.

2.4. Study Selection. Abstracts of retrieved articles were independently screened by two investigators (Wang and Zhou). Articles that did not meet the inclusion criteria were excluded and the remaining articles were fully reviewed. Any disagreements were resolved by the third reviewer (Cao).

2.5. Data Items and Data Collection Process. A standardized data collection form was used to extract the following information: first author's name, study country, year of publication, number of participants, mean age of sample, proportion of women in the study population, HF type, proportion of $\mathrm{HF}$ reduced Ejection Fraction (HFrEF) or HF preserved Ejection Fraction (HFpEF) patients, mean Ejection Fraction (EF), proportion of New York Heart Association (NYHA) class III-IV patients, definition of frailty, duration of follow-up, method of frailty measurement, prevalence of frailty in the sample, endpoints with corresponding HRs and 95\% CIs, and confounding factors adjusted for. We adopted the adjusted HR if both adjusted and crude estimates were provided. The data extraction process was independently performed by two investigators (Wang and Zhou). Any disagreements were resolved by the third reviewer (Cao).

2.6. Risk of Bias in Individual Studies. Two reviewers (Wang and Zhou) independently assessed the quality of included articles using the Newcastle-Ottawa Scale (NOS) [16]. The quality assessment tool evaluates the bias risk of each study from three aspects: (1) selection of the exposed and unexposed study populations; (2) comparability between the two groups; (3) outcome measurements. The maximum of these three dimensions is 4, 2, and 3, respectively. Any disagreements were resolved by the third reviewer (Cao).
2.7. Statistical Analysis. Review Manager 5.3 software was used for all data analysis. Only studies that used validated methods to assess frailty and provided HRs and 95\% CIs for the mortality of older HF patients were included in the meta-analysis. Statistical heterogeneity among studies was assessed by Cochran's $\mathrm{Q}$ test and $\mathrm{I}^{2}$ statistics, which indicate the percentage of total variation between studies due to heterogeneity rather than chance. If a low heterogeneity was observed $\left(\mathrm{I}^{2}<50 \%\right.$ or $\left.\mathrm{P}>0.10\right)$, we pooled the reported HRs using a fixed-effect model with the generic inverse variance method [17]. Otherwise, all HRs were pooled using a random-effects model via the same method. Potential publication bias was evaluated using the funnel plot [18].

\section{Results}

3.1. Study Selection. The flow chart of the literature retrieval is shown in Figure 1. After reviewing the full-text articles, 23 studies were excluded as they included nonelderly subjects ( $\mathrm{n}$ $=12$ ) or did not report HR ( $n=11)$. Ultimately, 10 prospective cohort studies were included in the systematic review [19-28].

3.2. Study Characteristics. Table 1 summarizes the characteristics of the ten studies included in the systematic review. Four studies were conducted in Italy $[20,22,26,28]$, three in the USA $[19,25,27]$, and three in Spain $[21,23,24]$. In total, the studies included 3033 elderly patients with HF. The sample sizes of the included articles were relatively small with 2 of the studies including less than 100 participants [19, 27]. Nine studies reported a mean age ranging from 74.9 to 85.2 years. One study failed to report the mean age. The majority of articles had almost equal male: female ratios, with only one article reporting a smaller number of women (28.81\%) [19]. In the included studies, the shortest follow-up period was 30 days and the longest was 20 years. The prevalence of frailty in older HF patients ranged from $25.4 \%$ to $76 \%$.

3.3. Frailty Assessment. Eight studies were classified as "Physical Frailty" as they primarily used physical frailty assessment tools such as the Fried Frailty Phenotype (FP) measurement $[19,21,23,24,27]$, portions of the five FP indicators (e.g., gait speed or handgrip strength) $[25,28]$, or the Short Physical Performance Battery (SPPB) [26]. Two studies were classified as "Multidimensional Frailty" as they used a multidimensional frailty measurement tool: Frailty Staging System (FSS) $[20,22]$, which evaluated multiple domains of elderly functioning.

3.4. Main Findings of the Eligible Studies. Table 2 highlights the main study findings. The association between frailty status and all-cause mortality was investigated in eight studies [19-24, 26, 28]. Three studies investigated the impact of frailty status on all-cause hospitalization $[25,27,28]$ and two studies investigated the effect of frailty on HF-related hospitalization $[27,28]$. The effects of frailty on readmission [23], 1-year readmission [24], 1-year readmission for HF [22], all-cause hospitalization or death [27], incidental functional limitations [23], and 30-day functional decline [24] were also investigated in some of the studies. 


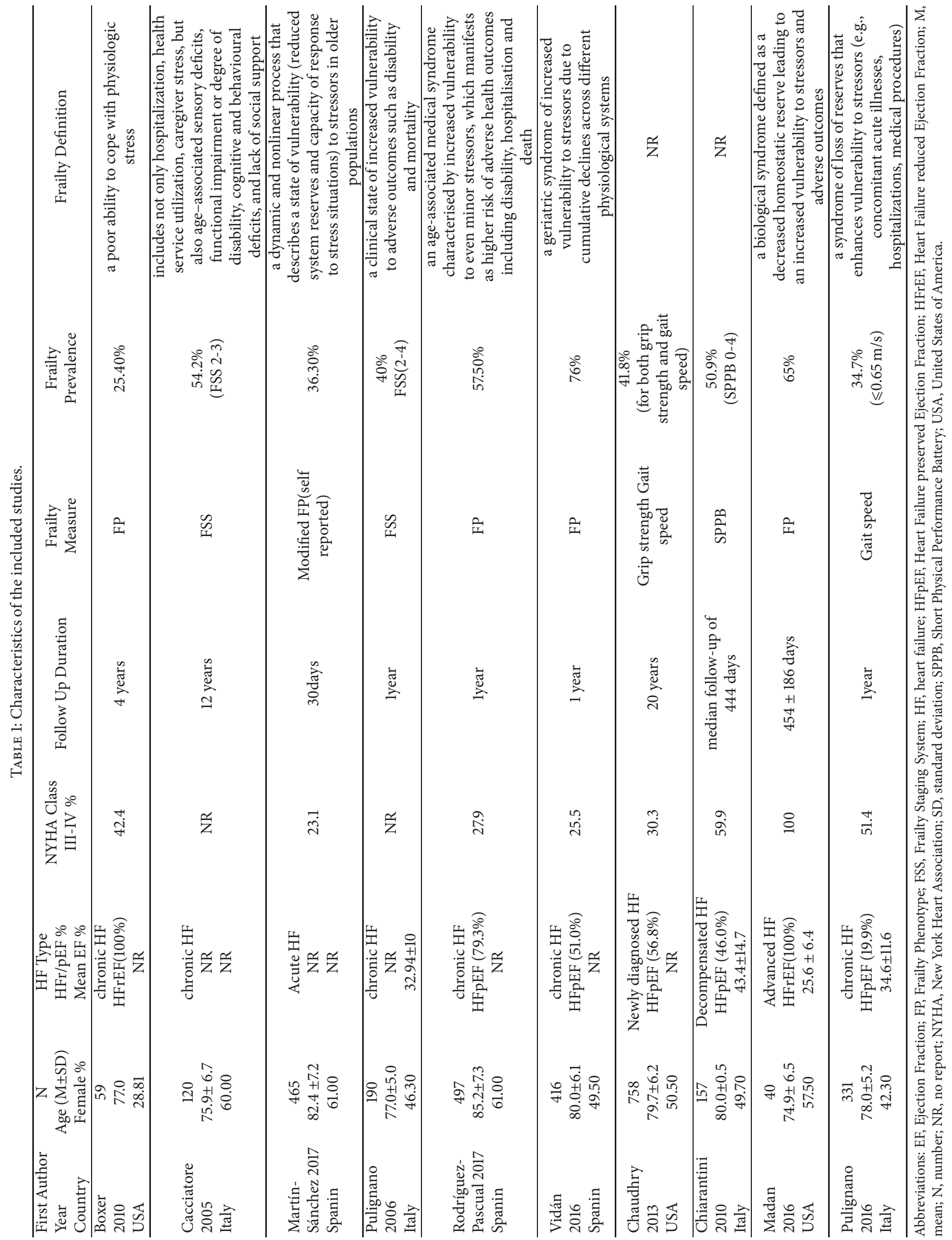




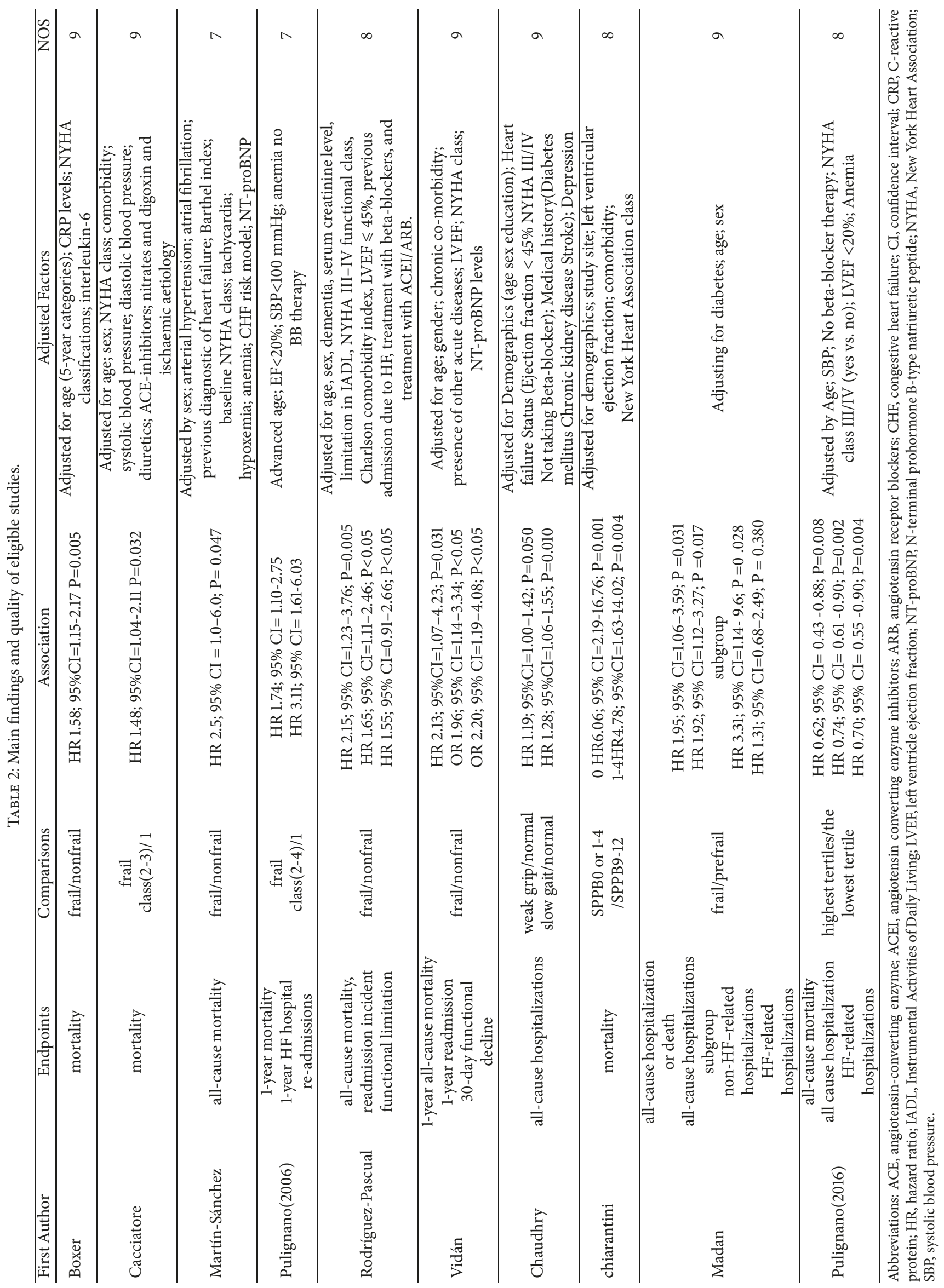




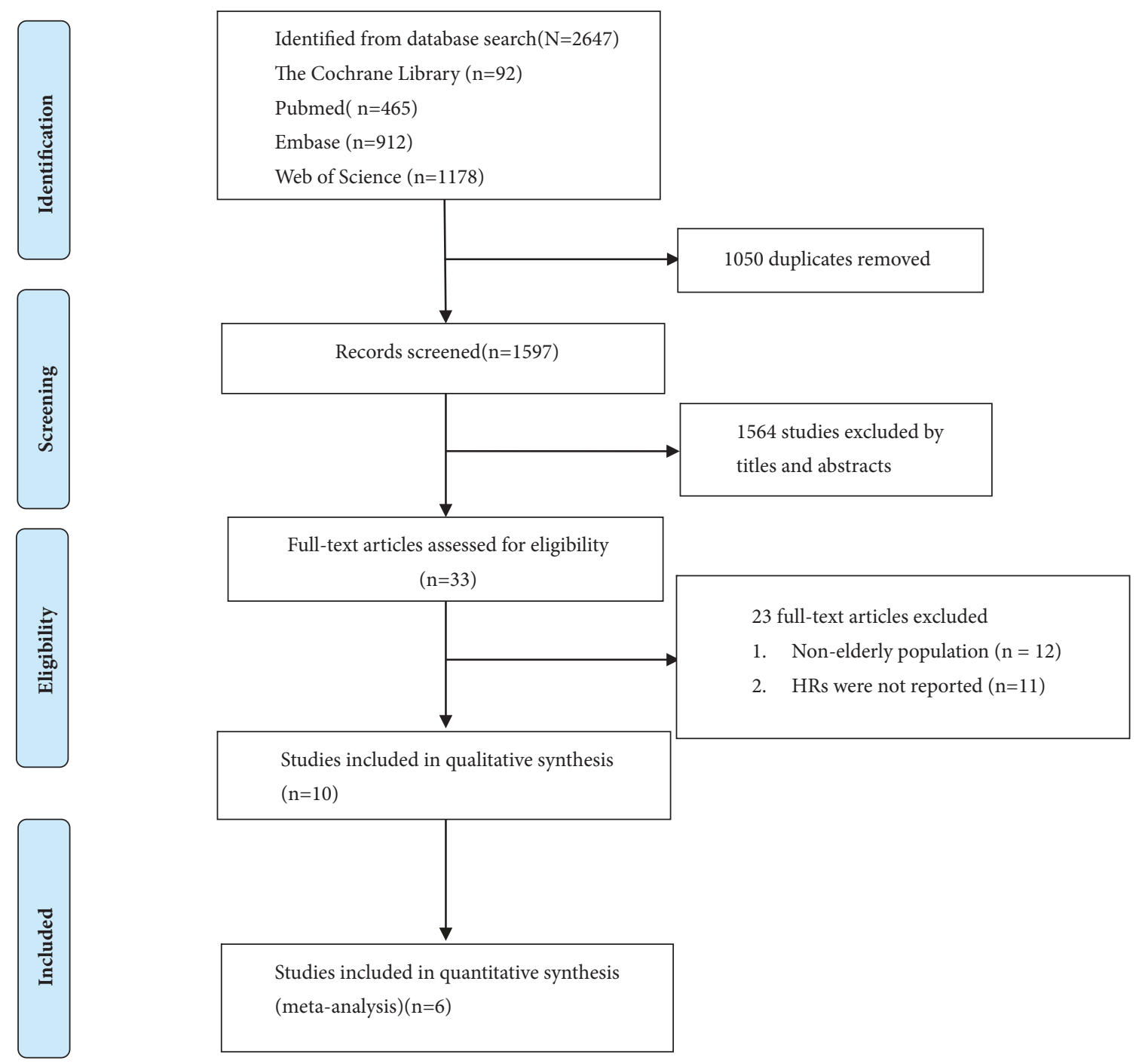

FIGURE 1: Literature screening process.

Detailed confounding factors adjusted for estimates of HRs are shown in Table 2. Well-characterized confounding factors include age $(n=9)$, sex $(n=7)$, NYHA class $(n=7)$, EF $(n=6)$, beta-blocker therapy $(n=4)$, and systolic blood pressure $(n=3)$.

3.5. Association between Frailty and Mortality in HF Patients. Eight studies reported the adjusted HRs for frailty for all-cause mortality in older HF patients [19-24, 26, 28]. Two studies were excluded due to the assessment tool for frailty and comparison of mortality rates among different frailty-status groups, leaving six articles that were eventually included in the meta-analysis [19-24]. Figure 2 depicts the summary effect estimated using the fixed-effects model. The pooled HR showed that, for older patients with HF, frailty was associated with a $70 \%$ higher risk of future mortality events (summary HR: 1.70, 95\% CI: 1.41-2.04). $\mathrm{I}^{2}=0$ (P<0.00001), suggesting low statistical heterogeneity among the studies included in the meta-analysis.
3.6. Methodological Quality Assessment. The quality assessment of the included studies is described in Table 2. For quality assessments, four studies were considered as having mild cohort selection bias as they were designed as multicenter studies $[21,23,26,28]$. One study was regarded as having high outcome bias, as it did not describe the outcome of the loss or evaluation methods of the endpoints [22]. One study was regarded as having mild outcome bias due to the short followup period [21].

3.7. Evaluation for Publication Bias. Obvious asymmetrical pattern is observed in Figure 3, indicating that potential publication bias existed across the studies.

\section{Discussion}

4.1. Frailty in Older HF Patients. This study showed that the prevalence of frailty in older patients with HF ranged from $25.4 \%$ to $76 \%$, similar to the $15 \%$ to $74 \%$ range in 


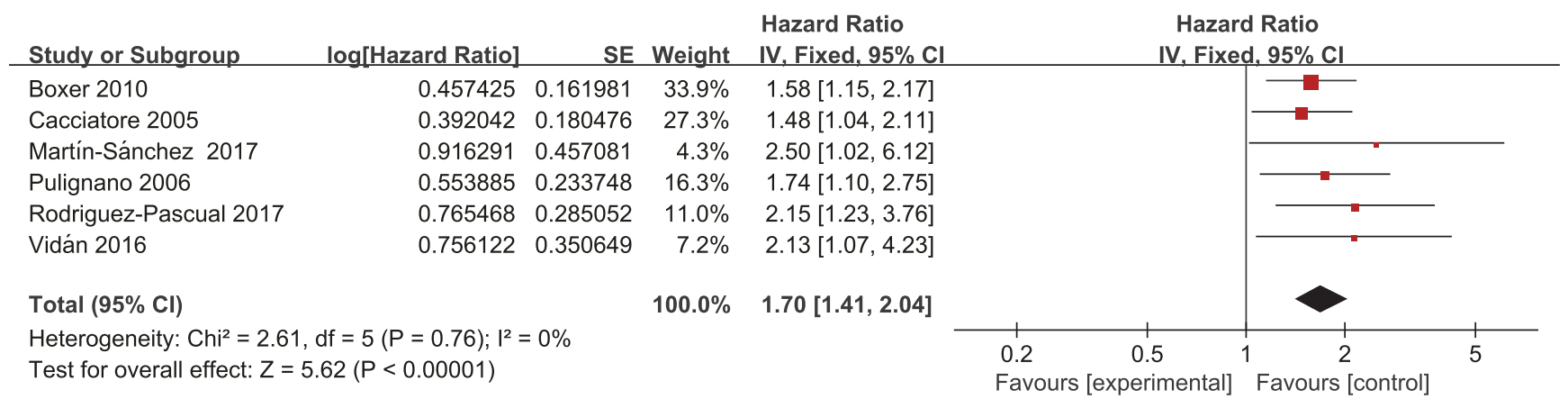

FIGURE 2: Forest plot of the HR for frailty for all-cause mortality in older HF patients.

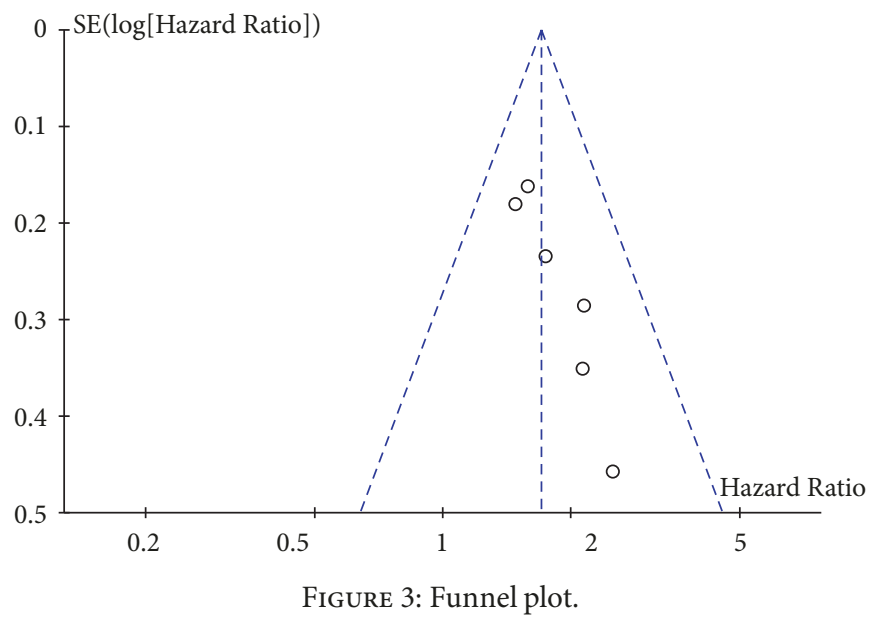

HF patients described in previous studies [29]. According to Fried's definition, the prevalence of frailty in the elderly community is $14 \%$ [9]. Thus, this study confirmed that the prevalence of frailty in older patients with HF is significantly higher than normal elderly population. Frailty was also an independent risk factor for HF in elderly patients. Compared to nonfrail older adults, the risk of HF in severely frail elderly patients increased by $88 \%$ [30]. Thus, it can be considered that $\mathrm{HF}$ and frailty are interrelated. HF increases the likelihood of becoming frail and frailty increases the risk of HF.

In addition, fatigue, weakness, and activity intolerance are common manifestations of $\mathrm{HF}$ and frailty [31, 32]. The underlying mechanisms of clinical similarities and coexistence may be the common pathological pathways involved in the two syndromes, including inflammatory processes and endocrine, metabolic, and autonomic dysfunction. The inflammatory process is the most critical of the aforementioned pathways $[33,34]$. Inflammation is known to play an important role in the development of cardiovascular diseases, including HF. Tissue depletion and cardiac cachexia are associated with elevated interleukin-1 (IL-1), interleukin-6 (IL-6), C-reactive protein (CRP), and tumor necrosis factor- $\alpha$ (TNF$\alpha)$ [32]. In addition, studies have found that inflammation is related to an increased incidence of frailty, and the levels of IL-6, CRP, TNF- $\alpha$, and other inflammatory markers among frail and elderly subjects were elevated [33, 34]. Elevated levels of inflammatory markers, particularly TNF- $\alpha$ and its soluble receptors, may lead to decreased muscle mass and strength by promoting catabolic processes in muscle cells. The loss of muscle mass is an important component of frailty. Results from Women's Health and Aging studies revealed an increased risk of frailty with an increased number of concurrent inflammatory diseases, further validating the inflammatory nature of frailty [33].

The exact mechanisms of frailty in older patients with $\mathrm{HF}$ remain unclear. It is likely that common pathophysiological processes between the two diseases reinforce each other through complex cellular and molecular mechanisms.

4.2. Frailty and Prognosis in Older HF Patients. We found that frailty was associated with increased adverse outcomes such as hospitalizations and mortality in older patients with HF. In addition, frailty also leads to a decline in the quality of life of older patients with $\operatorname{HF}[35,36]$. Above all, it was evident that, as a reduced physical reserve and vulnerable geriatric syndrome, frailty has an important effect on the development and prognosis of older patients with HF. Thus, greater attention to the prognostic value of frailty in older patients with $\mathrm{HF}$ is required.

Similar to geriatrics, care for patients with HF should broaden the coverage of traditional disease-oriented models. Despite an emphasis on the integrated management of 
HF patients, the most commonly used tools for assessing functional capacity in HF patients are limited to those that assess patient disability including NYHA, cardiopulmonary exercise testing, and six-minute walk testing [7]. The above assessments do not reflect the prognostic value of frailty in older patients with HF. In addition, frailty had a higher prognostic value for $\mathrm{HF}$ patients compared to subjective NYHA assessments. It is worth noting that a study has shown that a frailty assessment is better than traditional HF indexes for predicting short-term (30 days) mortality of older HF patients, suggesting that frailty as a state of systemic metabolic disorders is more important than traditional specificity indicators of HF [37]. Comprehensive and accurate risk assessments will be beneficial for individualized treatment regimens and information shared decisionmaking. According to our results, frailty plays an important role in the management of older patients with HF. Because frailty is reversible, its early detection in patients with $\mathrm{HF}$ helps to actively implement effective interventions, including physical activity and nutritional supplements that improve frailty and HF prognosis.

Furthermore, for patients with advanced HF, cardiac surgery such as heart transplantation and left ventricular assist device implantation often leads to severe postoperative morbidity and mortality during disease treatment. Therefore, the risk stratification should be performed prior to operation by comprehensively evaluating the patient's tolerance and ability to benefit from surgery [31,33]. At present, the risk assessment system of cardiac surgery is based primarily on chronological age. However, chronological age did not fully reflect the true functional status of the elderly, which can result in an inaccuracy of risk assessments. Recently, related studies have shown that frailty can increase the risk of adverse outcomes following cardiac surgery in the elderly $[38,39]$. Thus, frailty may also represent an important tool for stratifying the risk of invasive treatments in patients with HF. In future cardiac procedures, frailty assessments must be incorporated into risk prediction models.

4.3. Frailty Assessment in Older HF Patients. Frailty is more prevalent in older patients with $\mathrm{HF}$, and the presence of frailty often suggests a poorer prognosis. Therefore, the early identification of frailty in older patients with HF is crucial. Currently, there is no recognized gold standard for frailty assessment and no frailty assessment tool has been specifically validated in the HF population [40]. The results of this study indicated that frailty assessment tools in older patients with HF are inconsistent and the assessment of physical frailty overlaps with, but is not identical to, frailty instruments that assess multidimensional frailty. In addition, it should be noted that although some studies used validated assessment tools, the assessment process was not rigorous. For example, in studies by Martín-Sánchez and colleagues [21], despite the use of the FP to assess frailty, indicators such as gait speed and handgrip strength were self-reported questions that greatly reduced the accuracy of the assessments. Two articles used FSS to evaluate frailty, but the frailty ratings differed [20, 22].

In summary, the frailty assessment of older patients with $\mathrm{HF}$ requires standardization and unification. Otherwise, the differences caused by measurement methods cannot truly reflect the nature of the patient's disease.

4.4. Limitations. The present systematic review and metaanalysis has some limitations: (1) Due to the different tools used to evaluate frailty, clinical heterogeneity was unavoidable. (2) The confounding factors adjusted in individual studies varied and some important indicators were not adjusted in some studies, including NYHA class. An inadequate adjustment of confounding factors may overestimate or underestimate the prognostic value of frailty in older HF patients. (3) Only six studies were included in the final meta-analysis and publication bias was observed. Therefore, the prognostic value of frailty for older HF patients may be overestimated. (4) Our study was not registered prior to implementation meaning publication bias may exist. The operational study steps were however strictly in accordance with PRISMA guidelines. (5) HF itself is a heterogeneous disease and the types of HF differed across the studies included in the meta-analysis. Therefore, it is worth noting that the heterogeneity of HF across the study subjects may have affected our estimates of the prognostic value of frailty in older patients with HF. (6) During the selection of included articles, only those published in English were included. Therefore, the study contains selection bias.

\section{Conclusions}

The results of the present systematic review and meta-analysis indicate that frailty is more prevalent in older patients with $\mathrm{HF}$ and that frailty increases the risk of death by $70 \%$. Therefore, frailty is an effective indicator of the prognostic evaluation of older HF patients and clinical medical staff should attach importance to the role of frailty assessments during HF management. However, it should also be noted that a lack of standardization and unification of the assessment of frailty remains. Further studies are required to fully uncover the underlying pathological relationship between frailty and poor prognosis in older HF patients and explore effective intervention procedures to improve frailty and optimize $\mathrm{HF}$ prognosis.

\section{Conflicts of Interest}

The authors have no funding or conflicts of interest to disclose.

\section{References}

[1] D. Mozaffarian, E. J. Benjamin, A. S. Go et al., "Heart disease and stroke statistics-2015 update: a report from the American Heart Association," Circulation, vol. 131, no. 4, pp. e29-e322, 2015.

[2] S. J. Goodlin, "Function, failure, and frailty," Journal of Cardiac Failure, vol. 16, no. 5, pp. 396-397, 2010.

[3] D. W. Kitzman, B. Upadhya, and G. Reeves, "Hospitalizations and prognosis in elderly patients with heart failure and preserved ejection fraction: time to treat the whole patient," JACC: Heart Failure, vol. 3, no. 6, pp. 442-444, 2015.

[4] R. U. Shah, V. Tsai, L. Klein, and P. A. Heidenreich, "Characteristics and outcomes of very elderly patients after first 
hospitalization for heart failure," Circulation: Heart Failure, vol. 4, no. 3, pp. 301-307, 2011.

[5] P. Ferrero, A. Iacovoni, E. D’Elia, M. Vaduganathan, A. Gavazzi, and M. Senni, "Prognostic scores in heart failure - Critical appraisal and practical use," International Journal of Cardiology, vol. 188, no. 1, pp. 1-9, 2015.

[6] A. M. Mello, R. Custureri, and A. Pilotto, "Novel instruments for risk stratification of elderly patients with cardiovascular diseases," Giornale di Gerontologia, vol. 63, no. 2, pp. 61-68, 2015.

[7] R. Jermyn and S. Patel, "The biologic syndrome of frailty in heart failure," Clinical Medicine Insights: Cardiology, vol. 8, pp. 87-92, 2014.

[8] M. Guglin, T. Patel, and N. Darbinyan, "Symptoms in heart failure correlate poorly with objective haemodynamic parameters," International Journal of Clinical Practice, vol. 66, no. 12, pp. 1224-1229, 2012.

[9] L. P. Fried, C. M. Tangen, J. Walston et al., "Frailty in older adults: evidence for a phenotype," The Journals of Gerontology. Series A, Biological Sciences and Medical Sciences, vol. 56, no. 3, pp. M146-M156, 2001.

[10] J. E. Morley, B. Vellas, G. Abellan van Kan et al., "Frailty consensus: a call to action," Journal of the American Medical Directors Association, vol. 14, no. 6, pp. 392-397, 2013.

[11] J. Afilalo, K. P. Alexander, M. J. Mack et al., "Frailty assessment in the cardiovascular care of older adults," Journal of the American College of Cardiology, vol. 63, no. 8, pp. 747-762, 2014.

[12] M. T. Vidan, D. Cimera, E. Sanchez-Garcia et al., "Prevalence of frailty in elderly non-dependent patients hospitalized for heart failure, and characteristics of frail patients," European Heart Journal, vol. 34, pp. P657-P657, 2013.

[13] S. Yamada, K. Kamiya, and Y. Kono, "Frailty may be a risk marker for adverse outcome in patients with congestive heart failure," ESC Heart Failure, vol. 2, no. 3, pp. 168-170, 2015.

[14] J. Afilalo, "Frailty in patients with cardiovascular disease: why, when, and how to measure," Current Cardiovascular Risk Reports, vol. 5, no. 5, pp. 467-472, 2011.

[15] M. Goldfarb, R. Sheppard, and J. Afilalo, "Prognostic and therapeutic implications of frailty in older adults with heart failure," Current Cardiology Reports, vol. 17, no. 11, article 92, 2015.

[16] A. Stang, "Critical evaluation of the Newcastle-Ottawa scale for the assessment of the quality of nonrandomized studies in metaanalyses," European Journal of Epidemiology, vol. 25, no. 9, pp. 603-605, 2010.

[17] R. DerSimonian and N. Laird, "Meta-analysis in clinical trials," Controlled Clinical Trials, vol. 7, no. 3, pp. 177-188, 1986.

[18] M. Egger, G. D. Smith, M. Schneider, and C. Minder, "Bias in meta-analysis detected by a simple, graphical test," British Medical Journal, vol. 315, pp. 629-634, 1997.

[19] R. Boxer, A. Kleppinger, A. Ahmad, K. Annis, D. Hager, and A. Kenny, "The 6-minute walk is associated with frailty and predicts mortality in older adults with heart failure," Congestive Heart Failure, vol. 16, no. 5, pp. 208-213, 2010.

[20] F. Cacciatore, P. Abete, F. Mazzella et al., "Frailty predicts longterm mortality in elderly subjects with chronic heart failure," European Journal of Clinical Investigation, vol. 35, no. 12, pp. 723-730, 2005.

[21] F. J. Martín-Sánchez, E. Rodríguez-Adrada, C. Mueller et al., "The effect of frailty on 30-day mortality risk in older patients with acute heart failure attended in the emergency department," Academic Emergency Medicine, vol. 24, no. 3, pp. 298-307, 2017.
[22] G. Pulignano, D. Del Sindaco, A. Di Lenarda et al., "Frailty is an independent prognostic variable in very elderly patients with heart failure," European Journal of Heart Failure, vol. 27, 2006.

[23] C. Rodríguez-Pascual, E. Paredes-Galán, A.-I. FerreroMartínez et al., "The frailty syndrome is associated with adverse health outcomes in very old patients with stable heart failure: A prospective study in six Spanish hospitals," International Journal of Cardiology, vol. 236, pp. 296-303, 2017.

[24] M. T. Vidán, V. Blaya-Novakova, E. Sánchez, J. Ortiz, J. A. SerraRexach, and H. Bueno, "Prevalence and prognostic impact of frailty and its components in non-dependent elderly patients with heart failure," European Journal of Heart Failure, vol. 18, no. 7, pp. 869-875, 2016.

[25] S. I. Chaudhry, G. McAvay, S. Chen et al., "Risk factors for hospital admission among older persons with newly diagnosed heart failure: Findings from the cardiovascular health study," Journal of the American College of Cardiology, vol. 61, no. 6, pp. 635-642, 2013.

[26] D. Chiarantini, S. Volpato, F. Sioulis et al., "Lower extremity performance measures predict long-term prognosis in older patients hospitalized for heart failure," Journal of Cardiac Failure, vol. 16, no. 5, pp. 390-395, 2010.

[27] S. A. Madan, N. Fida, P. Barman et al., "Frailty assessment in advanced heart failure," Journal of Cardiac Failure, vol. 22, no. 10, pp. 840-844, 2016.

[28] G. Pulignano, D. Del Sindaco, A. Di Lenarda et al., "Incremental value of gait speed in predicting prognosis of older adults with heart failure: insights from the IMAGE-HF study," JACC: Heart Failure, vol. 4, no. 4, pp. 289-298, 2016.

[29] I. Uchmanowicz, M. Łoboz-Rudnicka, P. Szelag, B. JankowskaPolańska, and K. Łoboz-Grudzień, "Frailty in heart failure," Current Heart Failure Reports, vol. 11, no. 3, pp. 266-273, 2014.

[30] H. Khan, A. P. Kalogeropoulos, V. V. Georgiopoulou et al., "Frailty and risk for heart failure in older adults: The health, aging, and body composition study," American Heart Journal, vol. 166, no. 5, pp. 887-894, 2013.

[31] E. Joyce, "Frailty in advanced heart failure," Heart Failure Clinics, vol. 12, no. 3, pp. 363-374, 2016.

[32] S. M. Joseph and M. W. Rich, "Targeting frailty in heart failure," Current Treatment Options in Cardiovascular Medicine, vol. 19, no. 4, 2017.

[33] I. Uchmanowicz, M. Lisiak, R. Wontor et al., "Frailty Syndrome in cardiovascular disease: Clinical significance and research tools," European Journal of Cardiovascular Nursing, vol. 14, no. 4, pp. 303-309, 2015.

[34] H. M. Phan, J. S. Alpert, and M. Fain, "Frailty, inflammation, and cardiovascular disease: evidence of a connection," American Journal of Geriatric Cardiology, vol. 17, no. 2, pp. 101-107, 2008.

[35] H. G. Buck and B. Riegel, "The impact of frailty on health related quality of life in heart failure," European Journal of Cardiovascular Nursing, vol. 10, no. 3, pp. 159-166, 2011.

[36] I. Uchmanowicz, M. Wleklik, and B. Jankowska-Polanska, "The impact of frailty syndrome on quality of life in chronic heart failure," European Journal of Cardiovascular Nursing, vol. 15, 2016.

[37] A. Pilotto, F. Addante, M. Franceschi et al., "Multidimensional Prognostic Index based on a comprehensive geriatric assessment predicts short-term mortality in older patients with heart failure," Circulation: Heart Failure, vol. 3, no. 1, pp. 14-20, 2010.

[38] S. R. Jha, M. K. Hannu, S. Chang et al., "The prevalence and prognostic significance of frailty in patients with advanced heart 
failure referred for heart transplantation," Transplantation, vol. 100, no. 2, pp. 429-436, 2016.

[39] D. H. Lee, K. J. Buth, B.-J. Martin, A. M. Yip, and G. M. Hirsch, "Frail patients are at increased risk for mortality and prolonged institutional care after cardiac surgery," Circulation, vol. 121, no. 8, pp. 973-978, 2010.

[40] J. McDonagh, L. Martin, C. Ferguson et al., "Frailty assessment instruments in heart failure: A systematic review," European Journal of Cardiovascular Nursing, vol. 17, no. 1, pp. 23-25, 2018. 


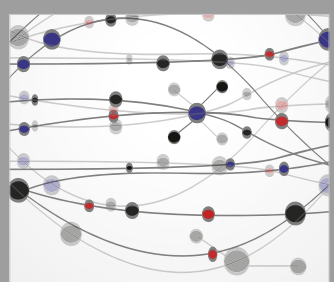

The Scientific World Journal
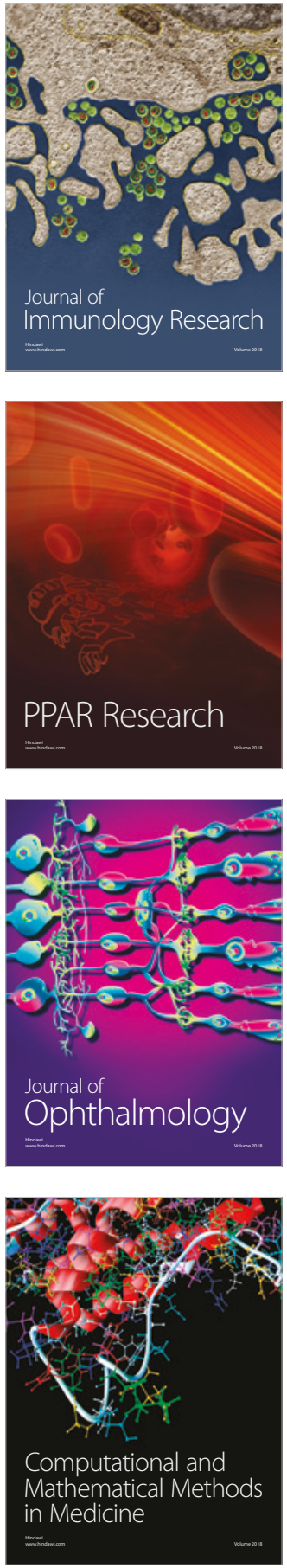

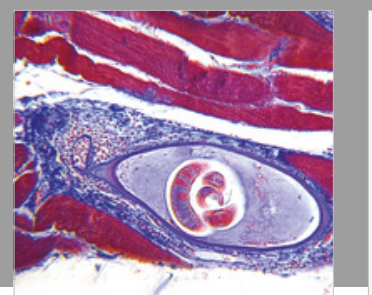

Gastroenterology Research and Practice

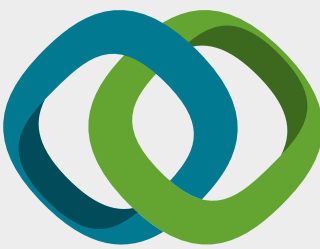

\section{Hindawi}

Submit your manuscripts at

www.hindawi.com
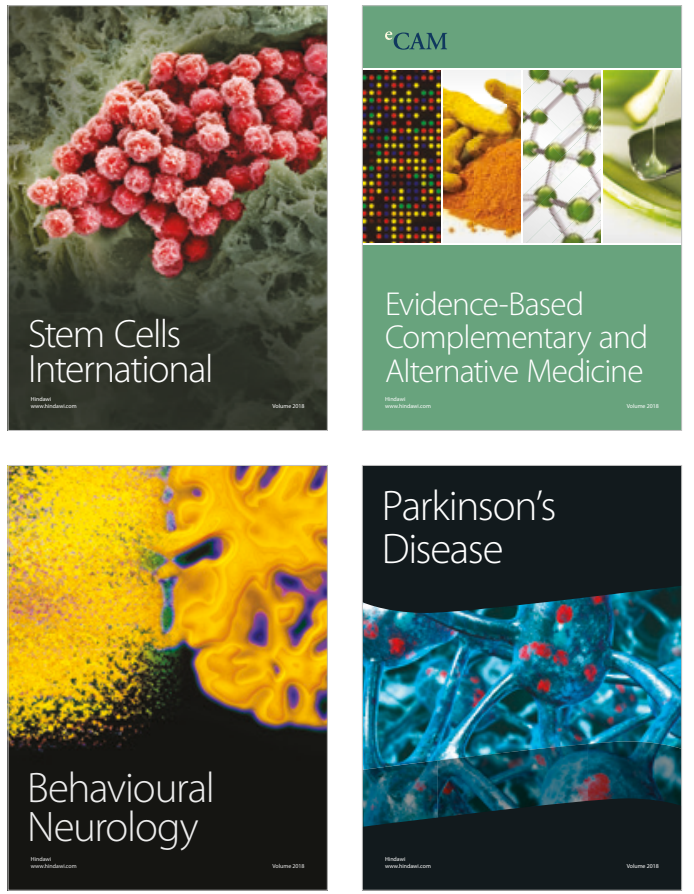

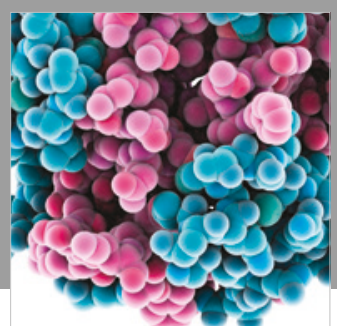

ournal of

Diabetes Research

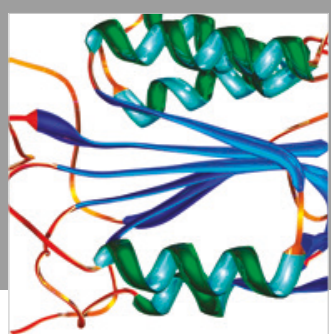

Disease Markers
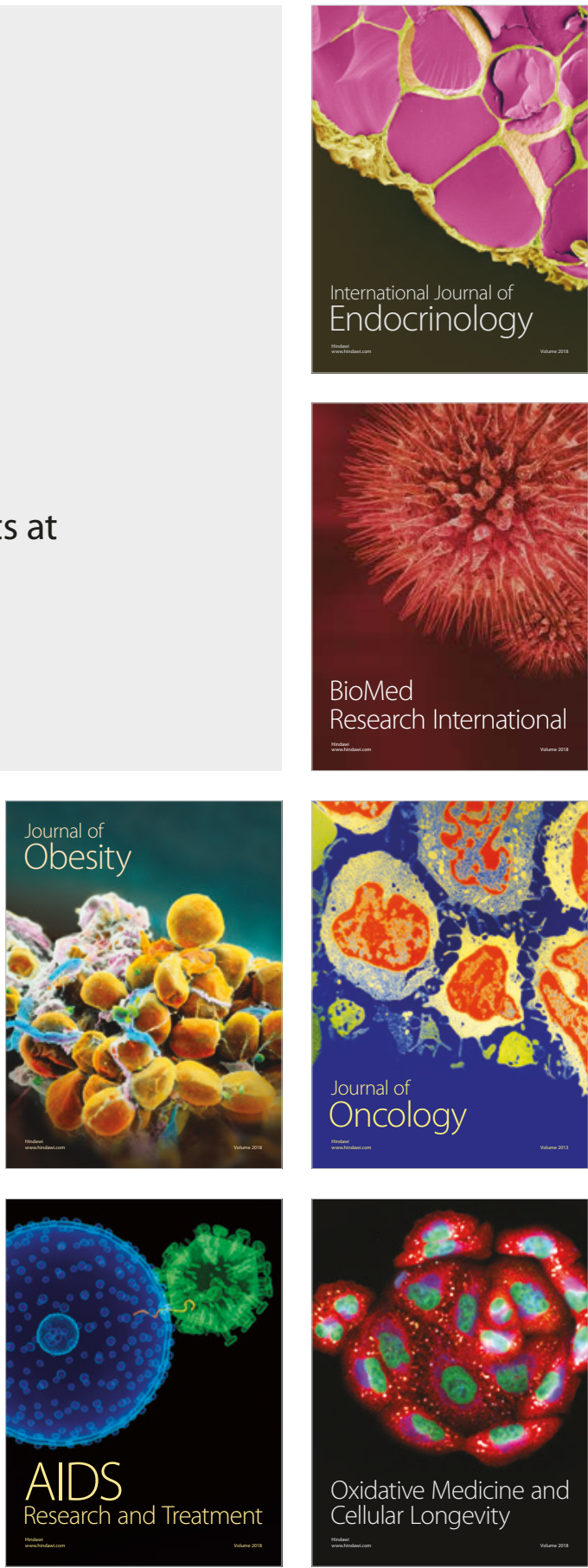